\title{
Intramuscular Loading dose versus Combined Intravenous \& Intramuscular Loading dose of Magnesium Sulphate in the Management of Eclampsia in a Tertiary Level Hospital of Bangladesh
}

\author{
S ROUF $^{\mathrm{a}}$, S AHMED ${ }^{\mathrm{b}}$, AAFRIN ${ }^{\mathrm{c}}$
}

Summary:

Background:Intramuscular loading dose of Magnesium Sulphate could be a suitable alternative for community intervention of the management of severe Pre- Eclampsia and Eclampsia. Objective: To compare the efficacy of the loading total IM regime of injection Magnesium Sulphate (MgSO4) with the standard loading combined IV and IM regime for termination \& prevention of recurrent convulsions in the treatment of Eclampsia. Methods: Total 200 patients were studied at Dhaka Medical College Hospital, Bangladesh from October 2012 to May 2013 where 100 patients were treated with loading IM regime (Case group) and 100 patients received standard loading combined IV \& IM regime (Control Group). The efficacy of both regimes was measured by the rate of recurrent convulsions. Results: No significant differences was observed in both groups in terms of age (23.03 \pm 3.90 vs $23.34 \pm 4.63$ years), parity (64\% vs $63 \%$ primi), gestational age (36.39 \pm 3.64 vs $36.13 \pm 3.95$ weeks), no of

Introduction:

Maternal mortality is a great concern for a developing country like Bangladesh. Bangladesh has to reach MDG5 by reducing the MMR to 1.47 / 1000 by $2015^{1}$. The fact is that the current MMR in Bangladesh is 1.94 / 1000 live births as on $2010,{ }^{2}$ is still one of the highest in south Asia. Near about 12000 to 15000 women die every year from maternal health related complications. ${ }^{3}$

Severe Pre Eclampsia (PE) and Eclampsia (E) are serious and relatively frequent complications of pregnancy and

a. Prof. Salma Rouf, Professor of Obs and Gynae, Dhaka Medical College and Hospital

b. Dr. Shatil Ahmed, Indoor Medical Office, Department of Obs and Gynae, Dhaka Medical College and Hospital

c. Ms. Ashiana Afrin, Management Trainee, UniMed and UniHealth Ltd.

Address of Correspondence: Prof. Salma Rouf, Professor of Obs and Gynae, Dhaka Medical College and Hospital, E-mail: salmarouf000@gmail.com

Received: 11 October, 2014

Accepted: 23 October, 2015 convulsions (5.28 \pm 3.21 vs $5.35 \pm 3.31$ times), mean diastolic blood pressure $(98.74 \pm 17.22$ vs $104.25 \pm 15.43 \mathrm{mmHg})$ and Glasgow Coma Scale (e"8, 96\% vs<8, 92\%). No significant differences observed between the two groups in mean convulsion to treatment interval (5.16 \pm 3.71 vs $4.95 \pm 3.12 \mathrm{hr}$ ) and convulsion to delivery interval (13.26 \pm 8.8 vs $13.95 \pm 8.46 \mathrm{hr})$. The recurrent convulsion rate was almost same in both groups (3\% vs 5\%, $ð^{2}=$ $0.521, P>0.05 n s)$. Case fatality was $2 \%$ in case group and $3 \%$ in control group (P $>0.05 n s)$. Conclusions: Loading total IM regime of $\mathrm{MgSO} 4$ is found as effective as the loading combined IV and IM regime in terms of control of convulsions and prevention of recurrent fits in Eclampsia. So it could be used by the field level workers before referral.

Key words: Magnesium sulphate, Pre-Eclampsia, Eclampsia management

(J Bangladesh Coll Phys Surg 2016; 34: 85-91)

is a major cause of poor pregnancy outcome, with a high maternal and perinatal mortality and severe obstetric morbidity in our country. Severe Pre-Eclampsia, often called a silent killer is characterized by high blood pressure accompanied by a high level of protein in the urine but without attention often go undetected and untreated and develops in to Eclampsia - the final and most severe phase of PE causing seizures, coma and even death of the mother and baby.

It is estimated that every year Eclampsia is associated with about 50,000 maternal deaths worldwide, predictably again most of which occur in the developing countries 2. The current incidence of Eclampsia are $0.04 \%$ to $0.1 \%$ in the United states and United kingdom, on the contrary with a much high rate in developing countries as great as $15 \%$ in some parts of Asia, Africa and Latin America. ${ }^{4}$ Eclampsia is the second most important cause of maternal death in Bangladesh contributing $20 \%$ of all 
maternal deaths due to obstetric complications ${ }^{2}$. Every day four mothers die due to Eclampsia contributing to 1500 maternal deaths / year. ${ }^{2}$ In resource poor developing countries the incidence of Eclampsia varies from 1 in 100 to 1 in $1700 .^{5}$

The BIRPERHT survey ${ }^{6}$ of maternal morbidity showed that national incidence of convulsion in pregnancy and puerperium was $7.9 \%$ and over 100000 women develop Eclampsia each year in Bangladesh. ${ }^{7}$ A number of studies have shown that case fatality of Eclampsia was very high in hospitals and is mainly due to delay in referral and management, long interval between onset of convulsion and reaching the hospitals. ${ }^{8}$

In many developing countries PE / Eclampsia is the leading cause of maternal mortality often claiming as many women's lives as Post Partum Hemorrhage (PPH), sometimes more. Unlike PPH prevention, management of Eclampsia still did not get priority in public and private health agenda due to lack of awareness and scarcity of clear policy and guidelines at the national level.Till now the management of Eclampsia is solely institutional based and there is no community level intervention. In the effort to detect all the Pre-Eclampsia before it become life threatening one approach is to take testing for hypertension (measuring BP) and proteinurea (checking urine for protein) in women in their homes rather than depending entirely on them to reach facilities .

In Bangladesh $71 \%$ of total deliveries happen in home by unskilled birth attendants and only $23 \%$ of women deliver under medical supervision ${ }^{9}$ and the rest have no access to obstetric care. ${ }^{9}$ Only $23.4 \%$ women in Bangladesh complete the recommended 4 ANC visits, 48.9\% have irregular ANC visits and 28.7\% do not seek any ANC during their entire pregnancies. ${ }^{2}$ So, there is a large unmet need for early detection of PE / E and many missed opportunities. According to national demographic surveys (DHS) unmet need for checking $\mathrm{BP}$ in pregnancy ranges from $13.9 \%$ in Indonesia to $53.1 \%$ in Bangladesh. ${ }^{10}$ As a result most Pre-Eclampsia remaining unrecognized until severe complications such as Eclampsia occur.

More over lack of availability of anticonvulsant druginjection Magnesium Sulphate $\left(\mathrm{MgSO}_{4}\right)$ at community and rural level, cost of Inj. $\mathrm{MgSO}_{4}$, lack of training of health care providers and supportive supervision also act as a barrier to ensure availability of Eclampsia prevention and management services throughout the nation. That is why mortality associated with PreEclampsia and Eclampsia shows little decline in more than $75 \%$ in low resource countries like Bangladesh. ${ }^{10} \mathrm{It}$ is the need of the time that a national guideline on using Magnesium Sulphate putting a loading dose at the lowest level by the field level health workers- FWV/ FWA (Family Welfare Visitor \& Family Welfare Assistant) at home before transferring women to facilities is very much required and is equally important in management perspective. If the patients could receive loading dose at home, this will prevent convulsion in severe PE and subsequent recurrent fits in Eclampsia during transfer to facilities and thereby decreases the chance of development of seizure related complicationsCVA, pulmonary oedema, unconsciousness, abruption etc.

Among the many anti convulsants Inj. Magnesium Sulphate topped the list and is recommended as anticonvulsant of choice after the Eclampsia trial in $1995^{11 .}$

There are 3 regimes of Magnesium Sulphate available for practicing. The popular one is combined IV and IM regime (Prichard) ${ }^{12}$ which is being used in Bangladesh, Intravenous regime (Zuspanregime) ${ }^{13}$ and Intramuscular regime ${ }^{14}$. Intramuscular regime is not being practiced in our country.

In Bangladesh Magnesium Sulphate was first introduced in $1996^{15}$ at Dhaka medical college hospital (DMCH) the largest tertiary Government teaching institute which has a separate Eclampsia ward with an average yearly admission of 700 - 800 Eclampsiapatients.After the two published pilot studies done at the same institute, ${ }^{15,16}$ a guideline was published by the Eclampsia working group in January $1998{ }^{17}$ and Magnesium Sulphate has been used routinely from the beginning of 1998, after the commencement of its production locally. According to the published guideline reduced dose schedule is recommended which is almost half of the dose described by the Collaborative Eclampsia trial. ${ }^{11}$ As the weight of the average Bangladeshi young women is light, this curtail dose appears to control convulsions effectively. Even it was found in a randomized controlled trial that only the initial loading dose was sufficient to arrest convulsions and prevent subsequent recurrences. ${ }^{18}$ 
It is very difficult to train the field level workers to calculate and prepare this IV and IM loading doses. Moreover they need to develop the skill of putting on IV cannulation. To remove the constrains of preparing and using IV and IM loading doses of $\mathrm{MgSO}_{4}$ at the community and rural level (by the field workers), we planned to conduct a case control study to compare the efficacy of loading IM regime and standard IV and IM regime of injection Magnesium Sulphate for Eclampsia to terminate convulsions and prevent its recurrence.

\section{Methods:}

This case control Quasi experimental study was conducted at the Eclampsia unit of Dhaka Medical College Hospital (DMCH) from October 2012 to May 2013. Dhaka Medical College hospital is one of the well reputed tertiary care teaching and training institute located at the centre of the capital. It has got a separate Eclampsia unit with round the clock emergency and intensive care facilities. The average yearly admission of Eclampsia patient is 700-800. During the study period a total of 200 patients were enrolled by purposive sampling.All the consecutive 100 antepartum and postpartum Eclampsia patients admitted in unit IV (Researcher's Unit) were taken as study population (Group A) and received 10gm IM loading dose of Inj. Magnesium Sulphate according to the following protocol.
All the consecutive 100 antepartum and postpartum Eclampsia patients admitted in other units were taken as control group (group-B) and received $10 \mathrm{gm} \mathrm{IV} \mathrm{and}$ IM loading dose (4 gm IV + 6 gm IM) of Inj. Magnesium Sulphate according to the following protocol.

Patients of both groups received an additional dose of 2.5 gm MgSo4 IV in diluted form if there was convulsion after $1 / 2$ an hour of loading dose which was considered as recurrent convulsion and put on maintenance therapy for 24 hours. The schedule of maintenance therapy was $2.5 \mathrm{gm}$ IM in alternate buttock at 4 hourly interval. Besides anticonvulsants patients of both groups were managed by the same protocol of Eclampsia management prepared by Eclampsia working group of Bangladesh. 17

The efficacy of both regimes was measured by the rate of recurrent convulsions.

Patients who received any anticonvulsants before hand from out-side were excluded from the study. The study was approved by departmental review board and ethical clearance was taken from the institutional ethical committee.

Results were expressed as incidence, mean \pm SD and proportion. Comparison between two groups was made with $\mathrm{x}^{2}$ analysis, $\mathrm{t}$ test as appropriate. $\mathrm{P}$ values of $<0.05$ was considered significant.

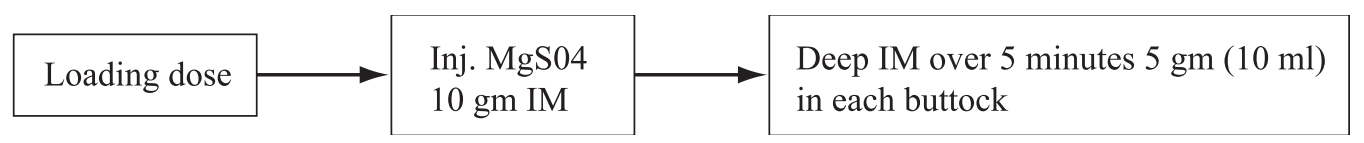

Fig.-1: Group A (study cases) Inj. $\mathrm{MgSO}_{4} \mathrm{IM}$ regime

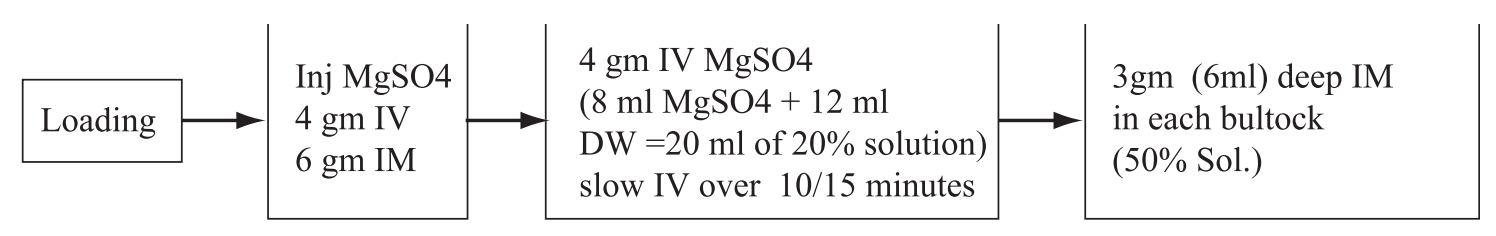

Fig.-2: Group B (Control cases) Inj. $\mathrm{MgSO}_{4} \mathrm{IV}$ and IM regime 


\section{Results:}

The study was conducted to compare the efficacy of the loading total IM regime of Injection Magnesium Sulphate with the standard loading combined IV and IM regime for termination of convulsion and prevention of its recurrence in the treatment of Eclampsia. A total of 200 patients were studied out of which 100 patients had received the loading total IM regime (Group-A or cases) and 100 patients were treated with the standard loading combined IV and IM regime (Group-B or controls). Sociodemographic characteristics of the patients, patients' profile, severity of the disease, patients' outcome and pregnancy outcome (both maternal and foetal) after recieving the loading dose of Magnesium Sulphate of both case and control groups were recorded. After data collection, processing and analysis, the following observations were obtained:

Table 1 described the characteristics of the patients and severity of the disease.In both the groups patients' profiles were almost the same. The average age of the case group was $23.03 \pm 3.90$ years and the control group was $23.34 \pm 4.63$ years. Most of the parameters which indicate severity of the disease on admissionrevealed no significant differences. The mean number of convulsions before admission was $5.28 \pm 3.21$ and 5.35 \pm 3.31 among the case and control groups respectively and other parameters like mean diastolic blood pressure (98.74 \pm 17.22 vs 104.25 $\pm 15.43 \mathrm{~mm} . \mathrm{Hg}$ ) and Glasgow Coma Scale (e”8, 96\% vs<8, 92\%) were almost the same.

No significant difference was observed in convulsion to treatment and convulsions to delivery intervals between the two groups (Table 2). The mean convulsion to treatment interval was $5.16 \pm 3.71 \mathrm{hr}$ in case group and $4.95 \pm 3.12 \mathrm{hr}$ in the control group. The mean convulsion to delivery interval was $13.26 \pm 8.80 \mathrm{hr}$ and $13.95 \pm 8.46 \mathrm{hr}$ respectively in the case and control groups. The recurrent convulsion rate was $3 \%$ in case group and $5 \%$ in the control group without any significant difference being observed $\left(ð^{2}=\right.$ 0.521 significance 0.718 ). Table. 3 showed the maternal and foetal outcome and the maternal death rate was $2 \%$ in the case group and $3 \%$ in the control group $\left(\mathrm{P}>0.05^{\mathrm{ns}}\right)$.

Table 3 also described the maternal outcome in relation to mode of delivery and faetal outcome. The rate of vaginal delivery (55\% vs 53\%), cesarean delivery (32\% vs $42 \%$ ), live birth (77\% and 79\%) and still birth (2\% vs $21 \%$ ) were found consistent among the two groups.

Table-I

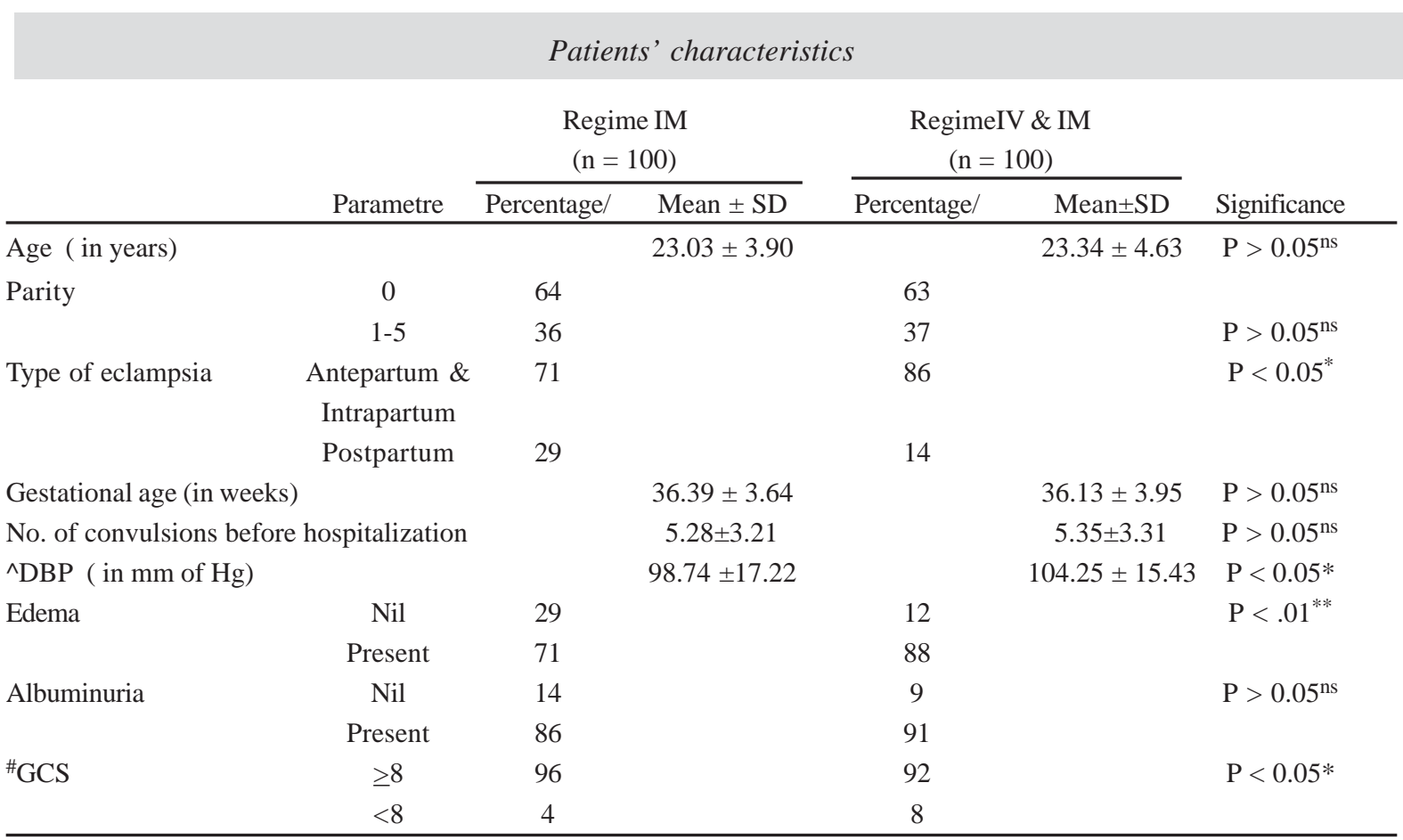

*/**: Significant $\wedge$ DBP :Diastolic Blood Pressurens : Not significant ${ }^{\#}$ GCS :Glasgow Coma ScaleSD : Standard deviation 


\section{Table-II}

\section{Outcome of two regimes in controlling Convulsions}

\begin{tabular}{|c|c|c|c|}
\hline Parametre & $\begin{array}{c}\text { Regime IM(n = 100) } \\
\text { Percentage/Mean } \pm \text { SD }\end{array}$ & $\begin{array}{c}\text { Regime IV \& IM( } \mathrm{n}=100) \\
\text { Percentage/Mean } \pm \text { SD }\end{array}$ & Significance \\
\hline Convulsion \& treatment interval (in hours) & $5.16 \pm 3.71$ & $4.95 \pm 3.12$ & $\mathrm{P}>0.05^{\mathrm{ns}}$ \\
\hline Convulsion \& delivery interval (in hours) & $13.26 \pm 8.80$ & $13.95 \pm 8.46$ & $\mathrm{P}>0.05^{\mathrm{ns}}$ \\
\hline Recurrence of convulsion after initiation of loading dose & 3 & 5 & $\mathrm{P}>0.05^{\mathrm{ns}}$ \\
\hline
\end{tabular}

Table-II

\section{Maternal and foetal outcome}

\begin{tabular}{|c|c|c|c|c|c|}
\hline \multirow[t]{2}{*}{ Parameter } & & & Regime IM & Regime IV \& IM & \multirow[b]{2}{*}{ Significance } \\
\hline & & & Percentage & Percentage & \\
\hline \multicolumn{6}{|l|}{ Maternal Outcome } \\
\hline \multirow[t]{3}{*}{ a) Mode of delivery } & $\wedge$ SVD & & 55 & 53 & $\mathrm{P}>0.05^{\mathrm{ns}}$ \\
\hline & Caesarian Section & & 32 & 42 & $\mathrm{P}>0.05^{\mathrm{ns}}$ \\
\hline & Ventouse / Forceps & & 12 & 5 & $\mathrm{P}>0.05^{\mathrm{ns}}$ \\
\hline b) Maternal death & & & 2 & 3 & $\mathrm{P}>0.05^{\mathrm{ns}}$ \\
\hline \multirow[t]{4}{*}{ Foetal Outcome } & Live birth & & 77 & 79 & $\mathrm{P}>0.05^{\mathrm{ns}}$ \\
\hline & & Low Birth Weight & 40 & 44 & \\
\hline & & Asphaxiated & 34 & 37 & \\
\hline & Stillbirth & & 22 & 21 & $\mathrm{P}>0.05^{\mathrm{ns}}$ \\
\hline
\end{tabular}

ns : Not Significant

$\wedge$ SVD :Spontaneous Vaginal Delivery

\section{Discussion:}

This quasi experimental study was carried out to determine whether the total intramuscular loading dose of Magnesium Sulphate is effective in controlling convulsions of Eclampsia patients and is comparable to the combined loading IV and IM regime in termination of convulsions and prevention of its recurrence. Magnesium Sulphate is the most recommended and commonly used medication throughout the world for treatment or prevention of seizure activity in patients with Pre Eclampsia and Eclampsia. In our country Magnesium Sulphate is available in 50\% solution of 2.5 $\mathrm{gm} / 5 \mathrm{ml}$ preparation. Forgiving $4 \mathrm{gm}$ IV as loading it has to be dilutedto make a $20 \%$ solution, making the calculation and preparation difficult for the field level workers. The combined IV and IM regime is most popularly practiced, The IM regime is not being practiced, but it is recommended to give $10 \mathrm{gm}$ IM loading dose before referral, if the provider is unable to give IV. ${ }^{19}$

The socio demographic characteristics of the patients in both the cases and the control groups of the present study (as discussed in Table: 1 ) revealed no significant differences in most aspects. Most of the study patients of both groups belonged to the age group of 21-25 years (52\% and 50\% respectively), were nulliparous (64\% and $63 \%$ respectively), came from poor socioeconomic group and house wife in profession (73\% to $72 \%$ respectively). So this study was done between two groups of almost similar types of patients and the result was not affected by the minor variations. The results were also found comparable to the other studies. ${ }^{18,} 20$

Regarding the pattern of antenatal care, most of the patients in both groups received irregular antenatal care 
(49\% and 37\% respectively) and a significant proportion had none (23\% and 24\%). This is consistent with the national findings which states that $28.7 \%$ women of Bangladesh do not seek any ANC, 26\% complete at least 4 ANC visits and the rest $45 \%$ of women have irregular ANC visits. ${ }^{9}$

The distribution of pattern of referral and the personnel who referred the patients to $\mathrm{DMCH}$ showed that majority of the patients came directly from home in both groups (49\% and $35 \%$ respectively), whereas quite a large number were also referred from district hospitals and private clinics (41\% and 46\% respectively) and a significant number were attended by doctors before being referred to DMCH (41\% and 57\%). This is much higher than the national findings which states that only $23 \%$ of births are attended by medical personnels. ${ }^{9}$ This could be explained by the fact that DMCH is the largest, most renowned and the most easily accessible tertiary level government institute of the country. So it receives complicated cases from all over the countries, usually referred by doctors. So training of the field level workers (like SBA, FWV and nurses) is urgently needed for early detection of $\mathrm{PE} / \mathrm{E}$ cases at home as well as offering loading dose of $\mathrm{MgSO}_{4}$ in convulsive women or severe PE cases at home or at lower facilities before transferring.

Table 1 also describes the parameters which indicate severity of the disease and has shown very subtle and negligible differences between the cases and the control groups. The mean number of convulsion before admission was $5.28 \pm 3.21$ in the case and $5.35 \pm 3.31$ in the control group. These findings were also found consistent with other studies. ${ }^{18,20}$

Table 2 shows the outcome of patients after receiving the loading dose in terms of recurrence of convulsions. The mean $( \pm \mathrm{SD}$ ) time required to initiate treatment in the cases and control groups were $5.55 \pm 5.07$ and 4.95 \pm 3.12 hours and to deliver after the onset of convulsions was $13.26 \pm 8.80$ and $13.95 \pm 8.46$ hours respectively between the two groups without any significant difference. In another prospective study carried out at DMCH, no significant difference was observed among the groups (group 1 only loading dose $n=202$ and group 2 standard regime $n=199$ ) in mean fit and treatment interval ( $6.88 \pm 5.26 \mathrm{vs} 7.12 \pm 4.29 \mathrm{hr}$ ) and fit and delivery interval $(11.35 \pm 10.22 \text { vs } 11 \pm 6.69 \mathrm{hr})^{18}$. So the present study findings were consistent and comparable with the previous study findings.
Regarding the recurrence of convulsions after receiving the loading dose, 3 patients of cases group (3\%) and 5 patients of control group (5\%) developed recurrent convulsions after initiation of the loading dose. The difference is not found statistically significant. Moreover recurrent convulsion rate was also found similar to the study done for comparing the efficacy of loading dose versus standard regime of Magnesium Sulphate ${ }^{18}$. So the loading total intramuscular regime appears to be equally effective like the loading combined IV and IM regime in preventing recurrence of convulsions in the treatment of Eclampsia.

Table 3 shows the case fatality and 2 patients of case group (2\%) and 3 patients of control group (3\%) died and among the 2 patients of case group 1 patient died undelivered. The case fatality rate was also comparable to the previous prospective comparativestudy ${ }^{18}$. As the difference is not statistically significant, the loading total intramuscular regime appears to be equally effective like the loading combined IV and IM regime in preventing maternal deaths in Eclampsia.

Regarding the maternal outcome in relation to mode of delivery and foetal outcome (live births and still births) in both 'cases' and 'control' groups of the present study observed no significant differences (Table.3).

From these above perspective it can be inferred that the loading total intramuscular regime is found as effective as the loading combined IV and IM regime in terms of control of convulsions and prevention of recurrence and seems to be at least equally effective in terms of managing pregnancy outcome and preventing case fatality if not better than IV and IM combined regime.

The strength of the study is that, this is the first study in Bangladesh which has been carried out to evaluate whether total intramuscular loading dose of Magnesium Sulphate is effective in controlling convulsions and prevention of its recurrence and is comparable to combined IV and IM regime. Though IV dose is comfortable for the patients and has immediate action, IM dose will be easy to administer for health workers of grassroot level.

Study shows that severe PE/E patients who received the loading doses before referral had reduced number of convulsions, more effective control of convulsions, shorter time to regain full consciousness, reduced maternal mortality and still birth rates ${ }^{21}$. So it is 
recommended to use loading doses at peripheral facilities and homebirths ${ }^{19},{ }^{21}$.Considering theclinical effectiveness demonstrated by the present study, ease of administration, ease of monitoring, easier availability and cost effectiveness, the loading total IM regime of Magnesium Sulphate appears to be equally preferable specially for the root level workers who will attend the Eclampsia patients or severe PE at home and can offer this treatment before referral. This will prevent further recurrence in Eclampsia and prevent development of fits in severe PE and thereby greatly improves the outcome by reducing convulsion treatment interval andpreventing seizure related complications. On the basis of the findings, integrated with the understanding from available literature, it will be recommended that a national guideline will be constructed for putting a loading total IM dose of Magnesium Sulphate at the lowest level by the field level workers before referral.

\section{References:}

1. Millennium Development Goals Midterm Bangladesh Progress Report 2007. Planning Commission GOB

2. Bangladesh Maternal Mortality and Health care survey 2010.

3. Millennium Development Goals Bangladesh Progress Report 2008. Planning Commission GOB

4. Regmi MC, Aggarwal A, Pradhan T, Rijal P, Subedi A and Duprety. Loading dose versus Standard regimen of Magnesium Sulphate in Eclampsia - a randomized trial. Nepal Med Coll J 2010; 12 (4): 244 — 247

5. Crowther CA. Eclampsia at Harare maternity hospital, an epidemiological study. S Aft. Med j 1985; 68: 927 929.[PubMed : 4081926]

6. The Bangladesh Institute of Research for promotion of Essential and Reproductive Health and Technologies ( BIRPERHT ). Proceedings of Dessiminassion workshop on maternal morbidity study. Hotel Sheraton, Dhaka, 1994.

7. Yasmin HA, Rahman MH, Chowdhury FK et al. Baseline survey for assessment of Emergency obstetric care services in Bangladesh. BIRPERHT, March $1995 ; 10$

8. Begum K, Hossain F. A study of 447 cases of eclampsia admitted in Dhaka Medical college Hospital. XV Asian Oceanic Cong obstetric gynaecol 1995 : 169 - 73
9. Bangladesh health and Demographic Survey 2011

10. Intervention for impact in essential obstetric and Newborn care. African Regional Meeting Report. 21 - 25 Feb.2011, Addis Abasa Ethiopia.

11. The Eclampsia Trial Collaborative group-Which anti convulsant for women with eclampsia? Evidence from the collaborative Eclampsia trial. Lancet 1995 : 345 ; $1455-1463$

12. Pritchard JA, Cunningham FG, Pritchard SA. The Parkland Memorial Hospital protocol for treatment of Eclampsia: Evaluation of 245 cases. Am J ObstetGynecol 1984; 148: 951-963.

13. Zuspan FP. Treatment of severe Pre Eclamsia and Eclampsia, ClinObstetGynecol 1996; 9: 945- 971.

14. Royal College of Obstetricians \&Gynaecologists. Life Saving Skills Manual. Essential Obstetrics and Newborn Care, Leverpool School of Tropical Medicine, World Health Organization, LATH 2007;22(ISBN 978-1 9047552-28-8)

15. Rouf S, Shamsuddin L. Khan JH. Magnesium Sulphate versus Diazepum in the management of eclampsia. Bangladesh J Obstet\&gynaecol 1996 ;Vol 11,1 : 1-14

16. L. Shamsuddin, S. Rouf. JH khan et al. Magnesium Sulphate versus Diazepam in the management of eclampsia. Bangladesh Medical Research Council Bulletin Aug 1998; Vol 24, 2: 43-8.

17. Eclampsia working group. Eclampsia in Bangladesh. A review and guideline Bangladesh J obstetriesGynaecology 1997, 12 No:1: 1- 27.

18. Begum M R, Begum A, Quadir E. Loading dose versus standard regime of Magnesium Sulphate in the management of Eclampsia ; A randomized trial . J. Obstet.Gynacol. Research June 2002; Vol. 28, No.3; 154-159.

19. WHO, UNFPA, UNICEF, The World Bank Group; Integrated Management of Pregnancy, Child birth, Postpartum and New born Care: A Guide for Essential Practices. WHO, Geneva 2006, $2^{\text {nd }}$ edition ( WQ 175)

20. Sultana N, Begum A, Begum K: Single loading dose of Magnesium Sulphate for control of Convulsion in Eclampsia. Journal of Bangladesh College of Physicians and Surgeons. May 2003, Vol 21, No-2, P 63-68

21. Begum MR, Begum A,Quadir E, Akhter S, L.Shamsuddin: Eclampsia: Still a Problem in Bangladesh. MedGenMed. MedScape General Medicine2004; 6(4):52. file://L: / Journal SR. 